Received: 2015.11.23 Accepted: 2016.01.10 Published: 2016.06 .07

\title{
Effects of Treatment of Asymptomatic Hyperuricemia on Graft Survival and Mortality in Kidney Transplant Recipients
}

Authors' Contribution: Study Design A Data Collection B Statistical Analysis C Data Interpretation D Manuscript Preparation E Literature Search F Funds Collection G
ABCDEf 1,2 Nikolaos Pagonas

BC 2 Samad Kor

DE 1,2 Felix S. Seibert

AE 1 Arnd Giese

AD 2 Walter Zidek

BD 3 Petra Reinke

BE 3 Nina Babel

ACE 1,2 Frederic Bauer*

ABDE 1,2 Timm H. Westhoff*
1 Medical Department I, Universitätsklinik Marien Hospital Herne, Ruhr-University of Bochum, Herne, Germany

2 Department of Nephrology, Charité - Campus Benjamin Franklin, Berlin, Germany 3 Department of Nephrology, Charité - Campus Virchow Klinik, Berlin, Germany
Corresponding Author: Source of support:

* Authors contributed equally to the work

Nikolaos Pagonas, e-mail: nikolaos.pagonas@marienhospital-herne.de Departmental sources

Background:

Material/Methods:

Results:

Conclusions:

MeSH Keywords:

Full-text PDF:
Hyperuricemia is very common after renal transplantation. It is associated with an increased risk of cardiovascular events and graft loss. To date, however, treatment is only recommended in symptomatic disease.

We included 503 adult patients who underwent kidney transplantation at the Charite-Universitätsmedizin Berlin in this retrospective study. Patients were followed up for up to 120 months. All-cause mortality, graft survival, changes in level of serum uric acid (SUA), and estimated glomerular filtration rate (eGFR) were analyzed.

At 12 months post-transplantation, 225 patients had a serum uric acid (SUA) level >7 mg/dl: 52 patients were treated with allopurinol, 37 with benzbromarone, and 136 patients received no medication for hyperuricemia (control). At 12 months, eGFR did not differ between groups $(p=0.15)$ but treated patients had higher SUA levels $(p<0.001)$ compared to the control group. SUA-lowering treatment was associated with a lower risk of allcause mortality $(p=0.013)$ and graft loss $(p=0.014)$ compared to controls. At 120 months, patients in the treatment group had lower SUA levels $(p=0.001)$ and higher eGFR $(p<0.001)$ compared to the control group.

Treatment of asymptomatic hyperuricemia was associated with a substantial benefit in patient and graft survival.

Graft Survival • Hyperuricemia • Kidney Transplantation

http://www.annalsoftransplantation.com/abstract/index/idArt/896821

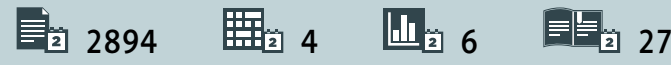




\section{Background}

Hyperuricemia is a common condition after renal transplantation. Whereas hyperuricemia has a prevalence of $10-15 \%$ in the general population, it may occur in up to $80 \%$ of renal transplant recipients [1]. The strikingly high prevalence after transplantation is predominantly caused by lower excretory function of the renal allograft and adverse effects of the immunosuppressive regimen, especially of calcineurin inhibitors (CNI). The minority of hyperuricemic patients develop symptomatic disease (e.g., symptoms of gout) in the course of time. However, the presence of hyperuricemia is associated with increased morbidity and mortality compared to transplanted patients without hyperuricemia [2]. Due to potential adverse effects of pharmacologic therapy, current guidelines do not recommend treatment of asymptomatic hyperuricemia in the general population [3]. There are no separate recommendations for patients with chronic kidney disease (CKD) or renal transplant recipients.

The effect of elevated serum uric acid level on the natural history of glomerular filtration rate (GFR) is controversial. There is increasing evidence that hyperuricemia is both a result of and a cause of reduction of GFR. In a seminal experimental study, Kang et al. [4] demonstrated that hyperuricemic rats develop more proteinuria and worse renal function after $5 / 6$ remnant kidney surgery than normouricemic rats. In an Asian cohort of 3850 subjects without preexisting renal disease, an elevation of uric acid accelerated the yearly decline in eGFR [5]. In a randomized prospective trial of 113 subjects with CKD and asymptomatic hyperuricemia, treatment with allopurinol led to a $6 \mathrm{ml} / \mathrm{min}$ increase in GFR [6], and indicated an effect of treatment on cardiovascular mortality. Consistently, both the NHANES I and the Framingham study demonstrated a positive correlation between uric acid and mortality $[7,8]$. In liver transplant recipients on a CNI-based immunosuppressive regimen, allopurinol improved renal function [9]. There are no data, however, on the effect of treatment of asymptomatic hyperuricemia on renal function and mortality after renal transplantation. We wondered whether treating hyperuricemia after renal transplantation would benefit patients with asymptomatic disease as well? In the present retrospective study, we analyze for the first time the effects of treatment of asymptomatic hyperuricemia on patient and graft survival.

\section{Material and Methods}

\section{Study design and population}

Patients who underwent kidney transplantation at 2 transplant centres of the university hospital Charité - Universitätsmedizin Berlin, Germany (Charité - Campus Benjamin Franklin-CBF and
Campus Virchow Klinikum-CVK) between 1997 and 2011 were screened for inclusion in this retrospective study. Patients were selected from the outpatient clinics of both transplantation centres. Inclusion criteria were renal transplantation between 1997 and 2011 and the presence of documented follow-up examinations, including laboratory and blood pressure values, in the outpatient clinic for at least 12 months after transplantation. We randomly selected 503 patients from the outpatient clinic. The group of patients treated for asymptomatic hyperuricemia were identified by a key word search using the terms "allopurinol, benzbromarone, uric acid, and hyperuricemia". The search yielded a total of 256 subjects. The control group was composed of 247 randomly assigned patients who were never treated for hyperuricemia during 10-year follow-up after transplantation. Combined pancreas/kidney transplantation and dialysis treatment at 4 weeks posttransplant were defined as exclusion criteria. At this time none of the patients had received serum uric acid (SUA)-lowering pharmacologic treatment. Data were collected at the following time points after transplantation: $1,3,6,12,36,60,84$, and 120 months. Subjects were generally divided into 2 groups depending on whether they received pharmacologic treatment for hyperuricemia at any posttransplant time point. Patients with a SUA $>7 \mathrm{mg} / \mathrm{dl}$ were regarded as hyperuricemic [10]. eGFR was calculated using the Modification of Diet in Renal Disease (MDRD) formula.

The clinical and research activities reported are consistent with the Principles of the Declaration of Helsinki and that of Istanbul as outlined in the 'Declaration of Istanbul on Organ Trafficking and Transplant Tourism".

\section{Statistical analysis}

Distribution of numeric data was analysed by the KolmogorovSmirnov test. In case of normal distribution, data are presented as mean \pm standard deviation; otherwise, as median and interquartile range (IR). Comparison of normally distributed numeric parameters was performed by 2-sided 2-sample $t$ tests. Comparison of categorical parameters was performed by Fisher's exact test in case of dichotomy (e.g., presence/absence of concomitant diseases, sex) and by the Pearson $\chi^{2}$ test in case of polychotomy (e.g., number of antihypertensives). Nonparametric tests were used for variables that were not normally distributed (Wilcoxon-Mann-Whitney test). Kaplan-Meier analysis was performed for survival outcomes. Cox proportional hazards model was used as univariate and multivariate analysis to estimate hazard ratios (HR) with $95 \%$ confidence intervals (CI) for the study outcomes. The multivariate model was adjusted for age at transplantation, sex, and baseline eGFR. Regression analysis was used for correlations of SUA and eGFR over time. Correlation of 2 parameters was assessed by Pearson's correlation analysis. The difference between regression lines was tested by analysis of covariance. According to previous trials, 


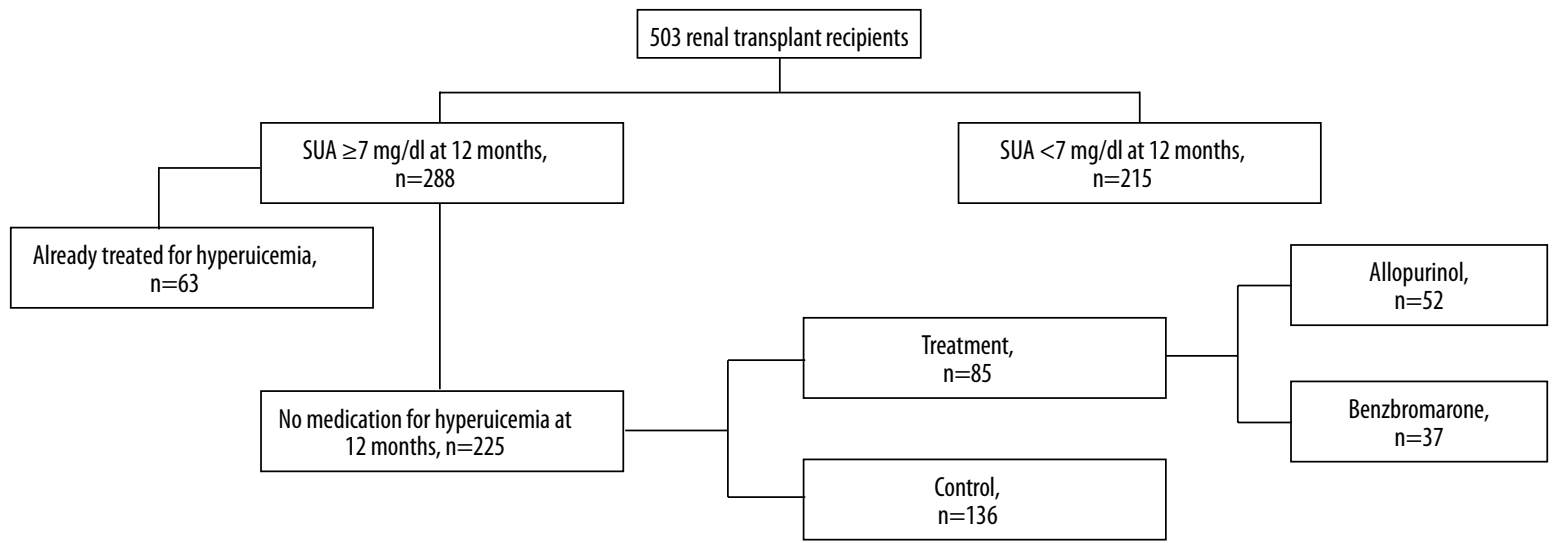

Figure 1. Trial profile.
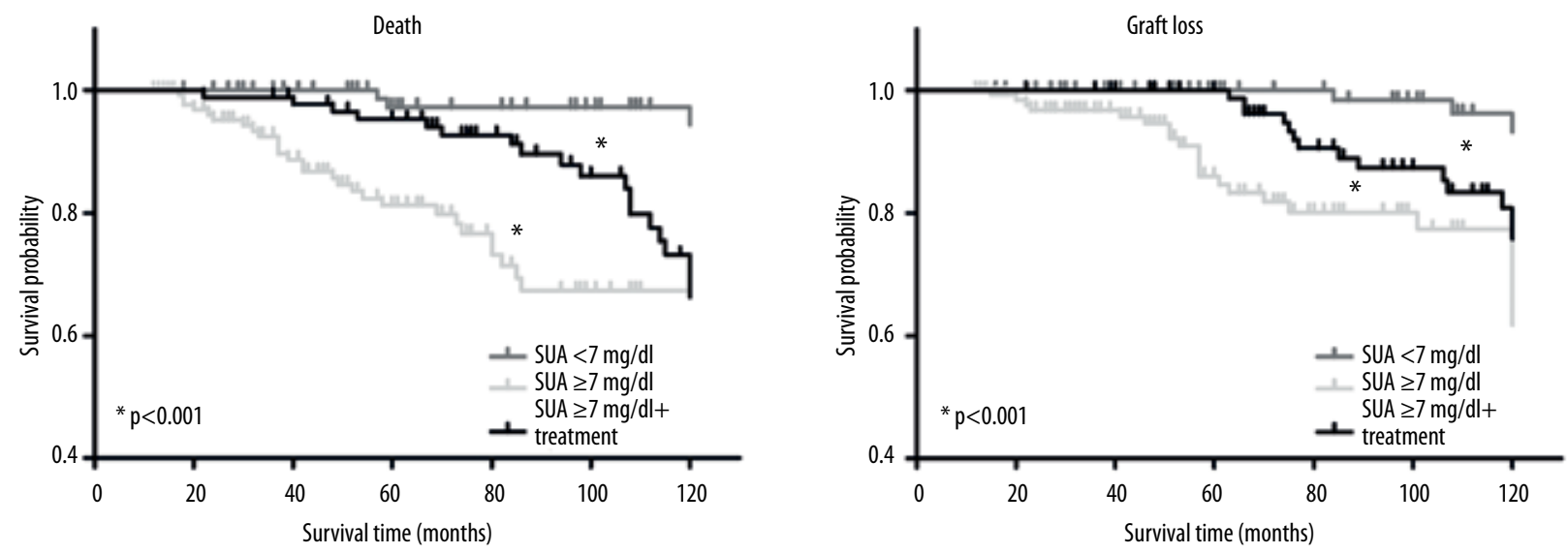

Figure 2. Kaplan-Meier survival curves based on serum uric acid (SUA) concentrations and the presence or absence of medication for hyperuricemia. Patients with lower SUA (SUA $<7 \mathrm{mg} / \mathrm{dl}$ ) showed lower rates of death and graft loss during follow-up $(p<0.001)$.

patients who returned to hemodialysis were supposed to have a GFR=10 ml/min $/ 1.73 \mathrm{~m}^{2}$ after graft loss [11]. For patients who died during the follow-up period, we imputed a GFR=0 $\mathrm{ml} /$ $\mathrm{min} / 1.73 \mathrm{~m}^{2}$ beyond death [12]. $\mathrm{P}<0.05$ was regarded as significant. All statistical analyses were performed using IBM SPSS Statistics, Version 20.0 (IBM Corp., Chicago, IL, USA).

\section{Results}

We included 503 subjects in the study according to the abovementioned criteria (Figure 1). Patients were followed up for up to 120 months with a mean follow-up of $81.2 \pm 35.6$ (range 12-120 months).

We initially performed a survival analysis among patients who were never treated for uric acid during the follow-up ( $n=247)$.
In order to explore effects of SUA on mortality, we divided patients into 2 groups based on the SUA $<7 \mathrm{mg} / \mathrm{dl}(\mathrm{n}=111)$ or SUA $\geq 7 \mathrm{mg} / \mathrm{dl}(\mathrm{n}=136)$ at 12 months. As presented in Figure 2, patients in the low SUA group demonstrated higher survival rates $(p<0.001)$ compared to those with SUA $\geq 7 \mathrm{mg} / \mathrm{dl}$ with or without treatment.

During follow-up, 256 patients (50.9\%) were treated with allopurinol $(n=128)$ or benzbromarone $(n=128)$ beginning at a mean of $27.0 \pm 29.9$ months after transplantation (range 0-119 months). One year posttransplant, regarded as a steady-state time point of renal function and immunosuppression, 288 (57.3\%) patients had a SUA $>7 \mathrm{mg} / \mathrm{dl}$. Among these, 63 (21.8\%) had already received medication for uric acid after transplantation (in the first 12 months) and were excluded from further analysis. The remaining 225 patients were divided into 2 groups based on intake of medication for uric acid at any time 
Table 1. Study population.

\begin{tabular}{|c|c|c|c|c|c|c|c|}
\hline \multirow[b]{3}{*}{ Female } & \multicolumn{7}{|c|}{ Total of patients with SUA $\geq 7$ mg/dl ( $n=225$ ) } \\
\hline & \multicolumn{2}{|c|}{ Total $(n=503)$} & \multicolumn{2}{|c|}{$\begin{array}{l}\text { No treatment } \\
\qquad(n=136)\end{array}$} & \multicolumn{2}{|c|}{$\begin{array}{c}\text { Allopurinol or } \\
\text { benzbromarone }(n=89)\end{array}$} & $\mathbf{p}$ \\
\hline & 188 & $(37.4 \%)$ & 47 & $(34.6 \%)$ & 29 & $(32.6 \%)$ & \multirow{2}{*}{0.77} \\
\hline Male & 315 & $(62.6 \%)$ & 89 & $(65.4 \%)$ & 60 & $(67.4 \%)$ & \\
\hline Age (years) & \multicolumn{2}{|c|}{$48.9 \pm 12.8$} & \multicolumn{2}{|c|}{$48.4 \pm 14.2$} & \multicolumn{2}{|c|}{$49.4 \pm 13.2$} & 0.60 \\
\hline Body mass index $\left(\mathrm{kg} / \mathrm{m}^{2}\right)$ & \multicolumn{2}{|c|}{$25.0 \pm 4.1$} & \multicolumn{2}{|c|}{$24.7 \pm 3.9$} & \multicolumn{2}{|c|}{$24.8 \pm 3.7$} & 0.82 \\
\hline Live donor transplantation & & $(12.5 \%)$ & 12 & $(9.1 \%)$ & 7 & $(8.0 \%)$ & 0.81 \\
\hline Time on dialysis (months, min-max) & \multicolumn{2}{|c|}{$59.8 \pm 42.6$} & \multicolumn{2}{|c|}{$61.4 \pm 45.9$} & \multicolumn{2}{|c|}{$60.1 \pm 36.3$} & 0.81 \\
\hline Donor sex male & 246 & $(58.2 \%)$ & 63 & $(56.8 \%)$ & 52 & $(65 \%)$ & 0.29 \\
\hline Donor age (years) & \multicolumn{2}{|c|}{$47.8 \pm 15.3$} & \multicolumn{2}{|c|}{$52.4 \pm 14.9$} & \multicolumn{2}{|c|}{$49.0 \pm 13.8$} & 0.09 \\
\hline Primary function & 194 & $(67.4 \%)$ & 99 & $(73.9 \%)$ & 58 & $(68.2 \%)$ & 0.44 \\
\hline Donor GFR $\left(\mathrm{ml} / \mathrm{min} / 1.73 \mathrm{~m}^{2}\right)$ & \multicolumn{2}{|c|}{$68.5 \pm 36.7$} & \multicolumn{2}{|c|}{$70.5 \pm 37.1$} & \multicolumn{2}{|c|}{$67.6 \pm 34.5$} & 0.68 \\
\hline \multicolumn{8}{|l|}{ Primary renal disease } \\
\hline Unknown & 95 & $(19.3 \%)$ & 29 & $(21.5 \%)$ & 24 & $(27.3 \%)$ & 0.34 \\
\hline Glomerulonephritis & 181 & $(36.0 \%)$ & 51 & $(37.8 \%)$ & 33 & $(37.5 \%)$ & 1.0 \\
\hline Interstitial nephritis & 42 & $(8.5 \%)$ & 6 & $(4.4 \%)$ & 10 & $(11.4 \%)$ & 0.06 \\
\hline Analgetic-induced nephropathy & 22 & $(4.5 \%)$ & 8 & $(5.9 \%)$ & 3 & $(3.4 \%)$ & 0.53 \\
\hline Cystic kidney disease & 66 & $(13.1 \%)$ & 10 & $(7.4 \%)$ & 7 & $(8.0 \%)$ & 1.0 \\
\hline Other hereditary kidney disease & 17 & $(3.4 \%)$ & 6 & $(4.4 \%)$ & 0 & $(0 \%)$ & 0.08 \\
\hline Nephrosclerosis & 24 & $(4.9 \%)$ & 13 & $(9.6 \%)$ & 2 & $(2.3 \%)$ & 0.05 \\
\hline Diabetes mellitus & 13 & $(2.6 \%)$ & 4 & $(3.0 \%)$ & 2 & $(2.3 \%)$ & 1.0 \\
\hline Amyloidosis & 5 & $(1.0 \%)$ & 0 & $(0.0 \%)$ & 2 & $(2.3 \%)$ & 0.16 \\
\hline Other & 12 & $(2.4 \%)$ & 5 & $(3.7 \%)$ & 2 & $(2.3 \%)$ & 0.71 \\
\hline \multicolumn{8}{|l|}{ Concomitant diseases } \\
\hline Hypertension & 482 & $(95.8 \%)$ & 125 & $(91.9 \%)$ & 86 & $(96.6 \%)$ & 0.17 \\
\hline Diabetes mellitus & 148 & $(29.4 \%)$ & 45 & (33.1\%) & 24 & $(27.0 \%)$ & 0.38 \\
\hline Coronary heart disease & 98 & $(19.6 \%)$ & 21 & $(15.6 \%)$ & 24 & $(27.05)$ & 0.04 \\
\hline Hyperlipidaemia & 180 & $(35.9 \%)$ & 35 & $(25.9 \%)$ & 32 & $(36 \%)$ & 0.14 \\
\hline
\end{tabular}

Epidemiological information, data on transplantation, cause of end-stage renal disease (ESRD), concomitant diseases, and cardiovascular risk factors of the study population. Numeric data are presented as mean and standard deviation. Numeric data were tested for statistically significant differences by unpaired t-tests. Categorical data (gender, live donation) were compared by Fisher's exact test. $\mathrm{P}<0.05$ was regarded statistically significant.

point during the follow-up period of 120 months. In the first group, 89 patients were treated with either allopurinol $(n=52)$ or benzbromarone $(n=37)$. The control group consisted of 136 patients who were not treated (Figure 1). Epidemiological information, data on transplantation, cause of end-stage renal disease (ESRD), concomitant diseases, and traditional cardiovascular risk factors are presented in Table 1 . Table 1 presents the findings of those subjects with a SUA concentration $>7 \mathrm{mg} / \mathrm{dl}$. Except for an increased incidence of coronary artery disease history in the treatment group $(p=0.04)$, there were no significant differences in patient baseline characteristics. Table 2 presents the immunosuppressive medication of the study population. The standard immunosuppressive regimen consisted of a calcineurin inhibitor ( $\mathrm{CNI}$; cyclosporine or tacrolimus), mycophenolic acid, prednisolone, and basiliximab if applicable. Mycophenolic acid was used more frequently in the control group in the early period after transplantation ( $p=0.02$ ). At 12 months after transplantation, cyclosporine trough levels did not differ between the treatment group $(120.9 \pm 34.8 \mathrm{ng} / \mathrm{ml})$ and the control group $(125.2 \pm 29.6 \mathrm{ng} / \mathrm{ml})$, 
Table 2. Immunosuppressive medication of the study population 4 weeks after transplantation.

\begin{tabular}{|c|c|c|c|c|c|c|c|}
\hline \multirow[b]{3}{*}{ Calcineurininhibitor (CNI) } & \multicolumn{7}{|c|}{ Total of patients with SUA $\geq 7$ mg/dl ( $n=225$ ) } \\
\hline & \multicolumn{2}{|c|}{ Total $(n=503)$} & \multicolumn{2}{|c|}{$\begin{array}{l}\text { No treatment } \\
\qquad(n=136)\end{array}$} & \multicolumn{2}{|c|}{$\begin{array}{c}\text { Allopurinol or } \\
\text { benzbromarone }(n=89)\end{array}$} & \multirow{2}{*}{$\begin{array}{c}p \\
0.82\end{array}$} \\
\hline & 412 & $(82.2 \%)$ & 122 & $(90.4 \%)$ & 79 & $(88.8 \%)$ & \\
\hline mTOR Inhibitors & 15 & $(3.0 \%)$ & 4 & $(3.0 \%)$ & 3 & $(3.4 \%)$ & 1.0 \\
\hline Mycophenolic acid & 372 & $(74.3 \%)$ & 116 & $(85.9 \%)$ & 65 & $(73 \%)$ & 0.02 \\
\hline Azathioprine & 40 & $(8.0 \%)$ & 10 & $(7.4 \%)$ & 10 & $(11.2 \%)$ & 0.35 \\
\hline Steroids & 489 & $(97.6 \%)$ & 134 & $(99.3 \%)$ & 87 & $(97.8 \%)$ & 0.56 \\
\hline
\end{tabular}
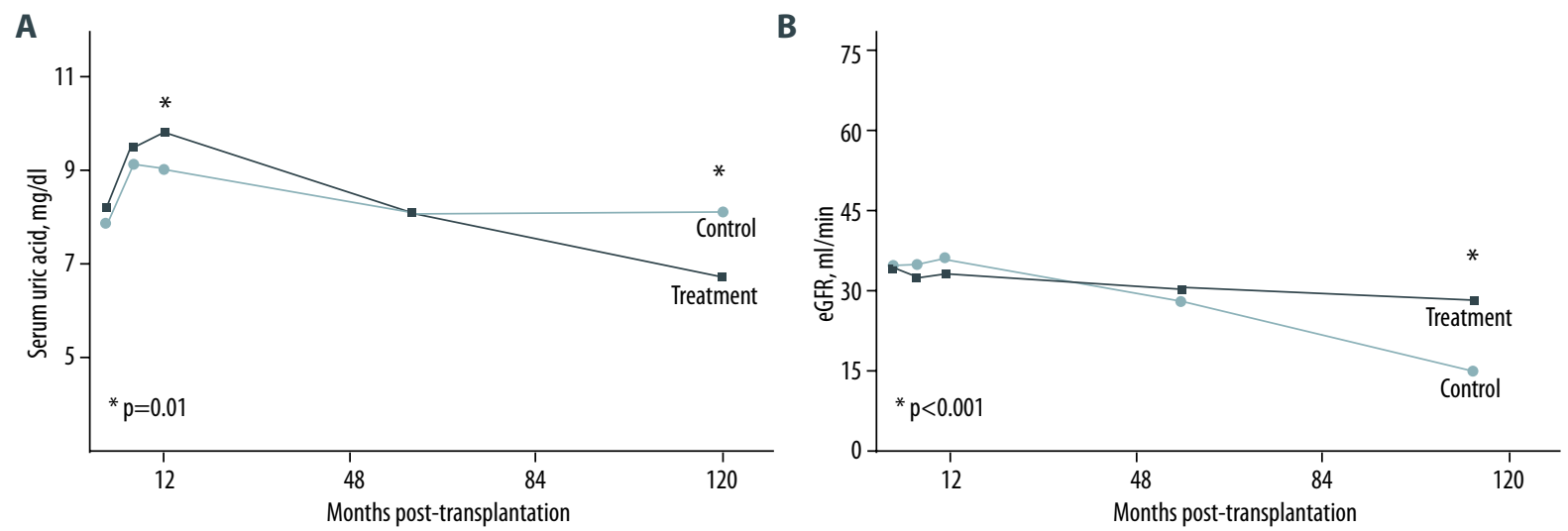

Figure 3. Changes of serum uric acid (SUA) and eGFR during follow-up. (A) Control patients had lower SUA at 12 months but higher SUA at 120 months compared to the treatment group ( $p=0.01$ ). (B) Changes in eGFR during the follow-up. No difference between groups at baseline. At 120 months, eGFR was significantly higher in the treatment group $(p<0.001)$.

$\mathrm{p}=0.46)$. No significant difference was detected for tacrolimus levels $(8.1 \pm 3.1$ vs. $7.2 \pm 3.0, p=0.19)$.

SUA and renal function were monitored throughout the follow-up period. At baseline, SUA and eGFR did not differ significantly between the groups ( $p>0.05$ each). After 10 years, patients in the treatment group had a higher eGFR compared to patients in the control group $\left(27 \mathrm{ml} / \mathrm{min} / 1.73 \mathrm{~m}^{2}\right.$, interquartile range $(I R)=10-44$ and $10 \mathrm{ml} / \mathrm{min} / 1.73 \mathrm{~m}^{2}, I R=0-10$ accordingly, $p<0.001$, Figure 3 ). Moreover, the annual loss of GFR, $\triangle G F R$, was significantly higher $(p=0.0015)$ in the control group $\left(-1.24 \mathrm{ml} / \mathrm{min} / 1.73 \mathrm{~m}^{2} /\right.$ year, IR $\left.-3.0,-0.2\right)$ compared to treated patients $\left(-0.35 \mathrm{ml} / \mathrm{min} / 1.73 \mathrm{~m}^{2} /\right.$ year, $\left.\mathrm{IR}-1.4,+1.3\right)$. Patients in the treatment group had a lower SUA at 10 years $(6.7 \pm 2.4 \mathrm{mg} / \mathrm{dl})$ in comparison to control patients $(8.1 \pm 1.2$ $\mathrm{mg} / \mathrm{dl}, \mathrm{p}=0.01$, Figure 3 ). Blood pressure and antihypertensive medication did not differ between the 2 groups at any time point (Table 3).

During the 10-year follow-up period, patients in the treatment group had better graft survival compared to the control group $(p=0.014)$. When adjusted for age at transplantation, sex, and
eGFR at 1 year posttransplant, the overall-mortality was lower in the treatment group (hazard ratio $(H R)=2.13,95 \%$ confidence interval $(\mathrm{Cl}): 1.17-3.87, \mathrm{p}=0.013)$. Both the incidence of the combined endpoint (death or graft loss) and the endpoint graft loss itself remained significantly lower in the treatment group in univariate analysis (Table 4). An adjustment for the baseline factors that differed between the groups (coronary artery disease and therapy with mycophenolic acid) in the Cox analysis did not influence the survival differences between the groups. The survival benefits for patients receiving therapy did not depend on the prescribed SUA-lowering agent (allopurinol and benzbromarone). Figure 4 presents the corresponding Cox regression survival curves.

To assess possible interactions between changes of SUA and eGFR, we performed regression analysis at the different time points. An inverse correlation between eGFR and SUA was seen for patients of both groups at 12 and 60 months. At both 12 and 60 months, the correlation coefficients were larger in women than in men in both groups. The slope of the line tended to be flatter at 60 months compared to 12 months in the treatment group $(\mathrm{eGFR}=-3.85 \times \mathrm{SUA}+71.0$ versus 
Table 3. Course of uric acid, graft function, blood pressure and antihypertensive medication during follow-up.

\begin{tabular}{|c|c|c|c|c|c|c|c|c|c|c|c|c|}
\hline & \multicolumn{3}{|c|}{$\begin{array}{l}4 \text { weeks posttransplant } \\
\text { (no treatment) }\end{array}$} & \multicolumn{3}{|c|}{$\begin{array}{l}1 \text { year posttransplant } \\
\text { (begin of treatment) }\end{array}$} & \multicolumn{3}{|c|}{$\begin{array}{c}5 \text { years } \\
\text { posttransplant }\end{array}$} & \multicolumn{3}{|c|}{$\begin{array}{l}10 \text { years } \\
\text { posttransplant }\end{array}$} \\
\hline & Control & Treatment & $P$ & Control & Treatment & $\mathbf{P}$ & Control & Treatment & $\mathbf{P}$ & Control & Treatment & $\mathbf{P}$ \\
\hline $\begin{array}{l}\text { SUA } \\
(\mathrm{mg} / \mathrm{dl})\end{array}$ & $7.5 \pm 2.4$ & $8.4 \pm 2.2$ & 0.034 & $8.6 \pm 1.3$ & $9.9 \pm 1.9$ & $<0.001$ & $8.1 \pm 1.7$ & $8.0 \pm 2.2$ & 0.88 & $8.1 \pm 1.2$ & $6.7 \pm 2.4$ & 0.01 \\
\hline $\begin{array}{l}\text { eGFR } \\
(\mathrm{ml} / \mathrm{min})\end{array}$ & $\begin{array}{c}32 \\
(20-48)\end{array}$ & $\begin{array}{c}30 \\
(20-41)\end{array}$ & 0.33 & $\begin{array}{c}37 \\
(21-47)\end{array}$ & $\begin{array}{c}31 \\
(23-40)\end{array}$ & 0.15 & $\begin{array}{c}26 \\
(10-47)\end{array}$ & $\begin{array}{c}29 \\
(22-41)\end{array}$ & 0.27 & $\begin{array}{c}10 \\
(0-10)\end{array}$ & $\begin{array}{c}27 \\
(10-44)\end{array}$ & $<0.001$ \\
\hline $\begin{array}{l}\text { Systolic BP } \\
(\mathrm{mmHg})\end{array}$ & $\begin{array}{c}140 \\
(130-150)\end{array}$ & $\begin{array}{c}140 \\
(130-150)\end{array}$ & 0.72 & $\begin{array}{c}130 \\
(124-140)\end{array}$ & $\begin{array}{c}131 \\
(122-140)\end{array}$ & 0.40 & $\begin{array}{c}130 \\
(120-136)\end{array}$ & $\begin{array}{c}130 \\
(120-140)\end{array}$ & 0.35 & $\begin{array}{c}120 \\
(116-145)\end{array}$ & $\begin{array}{c}130 \\
(120-138)\end{array}$ & 0.67 \\
\hline $\begin{array}{l}\text { Diastolic BP } \\
(\mathrm{mmHg})\end{array}$ & $\begin{array}{c}82 \\
(78-90)\end{array}$ & $\begin{array}{c}80 \\
(70-90)\end{array}$ & 0.08 & $\begin{array}{c}80 \\
(74-86)\end{array}$ & $\begin{array}{c}80 \\
(70-85)\end{array}$ & 0.48 & $\begin{array}{c}80 \\
(72-85)\end{array}$ & $\begin{array}{c}80 \\
(70-82)\end{array}$ & 0.19 & $\begin{array}{c}79 \\
(70-81)\end{array}$ & $\begin{array}{c}80 \\
(70-86)\end{array}$ & 0.74 \\
\hline $\begin{array}{l}\text { Average } \\
\text { number of } \\
\text { antihyper- } \\
\text { tensives }\end{array}$ & $\begin{array}{c}2 \\
(0-5)\end{array}$ & $\begin{array}{c}2 \\
(0-5)\end{array}$ & 0.69 & $\begin{array}{c}3 \\
(0-6)\end{array}$ & $\begin{array}{c}3 \\
(0-6)\end{array}$ & 0.50 & $\begin{array}{c}2 \\
(0-6)\end{array}$ & $\begin{array}{c}3 \\
(0-5)\end{array}$ & 0.08 & $\begin{array}{c}3 \\
(0-4)\end{array}$ & $\begin{array}{c}3 \\
(0-5)\end{array}$ & 0.94 \\
\hline
\end{tabular}

SUA (serum uric acid) is presented as means \pm standard deviation (normally distributed). eGFR and blood pressure values are presented as median and interquartile range (not normally distributed). The average number of medication is presented as median and range (in parenthesis). Statistical tests used for the individual parameters are presented in the statistics section. $P<0.05$ was regarded as statistically significant. eGFR - estimated glomerular filtration rate; BP - blood pressure.

Table 4. Cox regression analysis for study outcomes.

\begin{tabular}{|c|c|c|c|c|c|}
\hline Outcome & \multicolumn{2}{|c|}{ Treatment group $n=89$} & \multicolumn{2}{|c|}{ Control group $n=136$} & $P$ value \\
\hline \multicolumn{6}{|l|}{ All-cause mortality } \\
\hline Number of events & 19 & $(21.3 \%)$ & 28 & $(20.6 \%)$ & \\
\hline Univariate HR $(95 \% \mathrm{Cl})$ & & & 1.71 & $(0.95-3.08)$ & 0.07 \\
\hline Multivariate HR $(95 \% \mathrm{Cl})$ & & & 2.13 & $(1.17-3.87)$ & 0.013 \\
\hline \multicolumn{6}{|l|}{ Renal graft loss } \\
\hline Number of events & 14 & $(15.7 \%)$ & 25 & $(18.4 \%)$ & \\
\hline Univariate $\mathrm{HR}(95 \% \mathrm{Cl})$ & & & 2.22 & $(1.15-4.30)$ & 0.04 \\
\hline Multivariate HR $(95 \% \mathrm{Cl})$ & & & 2.32 & $(1.19-4.51)$ & 0.014 \\
\hline \multicolumn{6}{|l|}{ Renal graft loss or death } \\
\hline Number of events & 33 & (37.1\%) & 50 & $(36.8 \%)$ & \\
\hline Univariate $\mathrm{HR}(95 \% \mathrm{Cl})$ & & & 1.82 & $(1.17-2.84)$ & 0.007 \\
\hline Multivariate HR $(95 \% \mathrm{Cl})$ & & & 2.15 & $(1.38-3.37)$ & 0.001 \\
\hline
\end{tabular}

Number of events (in percentage of total) in each group during a mean of 75.5 months (6.3 years) follow-up. Univariate and multivariate hazard ratios with $95 \%$ confidence intervals $(\mathrm{HR}, 95 \% \mathrm{Cl})$ for study outcomes for control and treatment group (used as reference in Cox analysis). In the multivariate model adjustments were made for age, gender and eGFR at 12 months after transplantation. $\mathrm{HR}$ - hazard ratio; $\mathrm{Cl}-95 \%$ confidence interval.

eGFR=-2.24×SUA+49.1, p=0.09). In the subpopulation of women treated against uric acid, the slope was significantly flatter at 60 months compared to 12 months (eGFR=-5.22×SUA+78.9 versus eGFR $=-2.42 \times S U A+43.5$, respectively, $p=0.04$ ). The diagrams of the regression analyses are presented in Figures 5, 6 . 
A

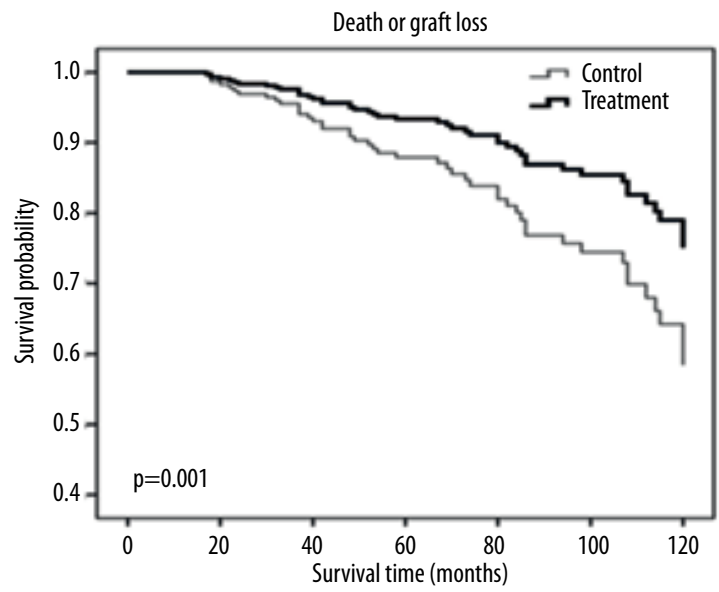

C

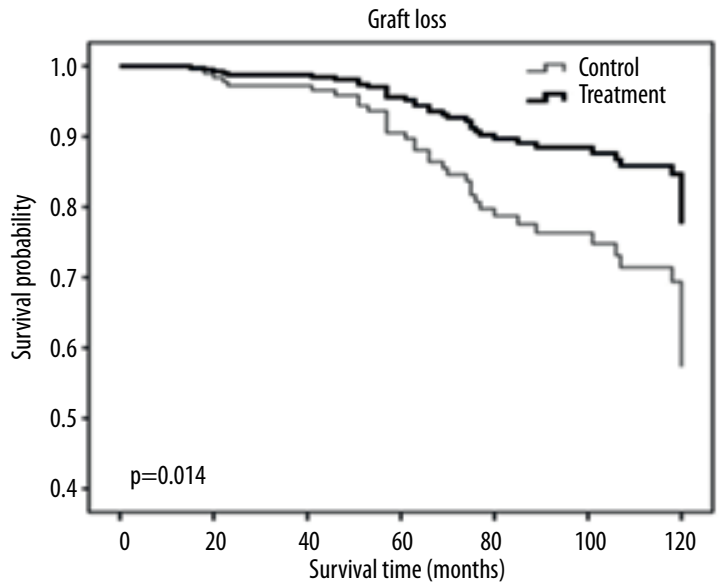

B

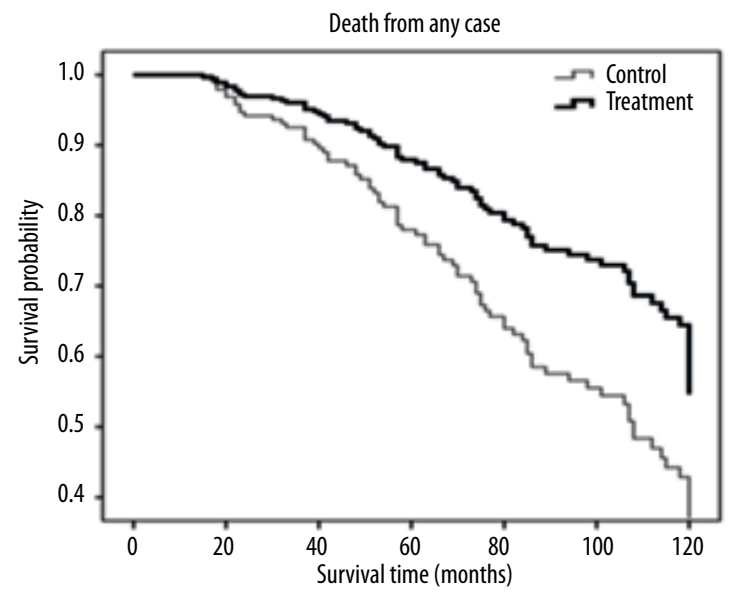

D

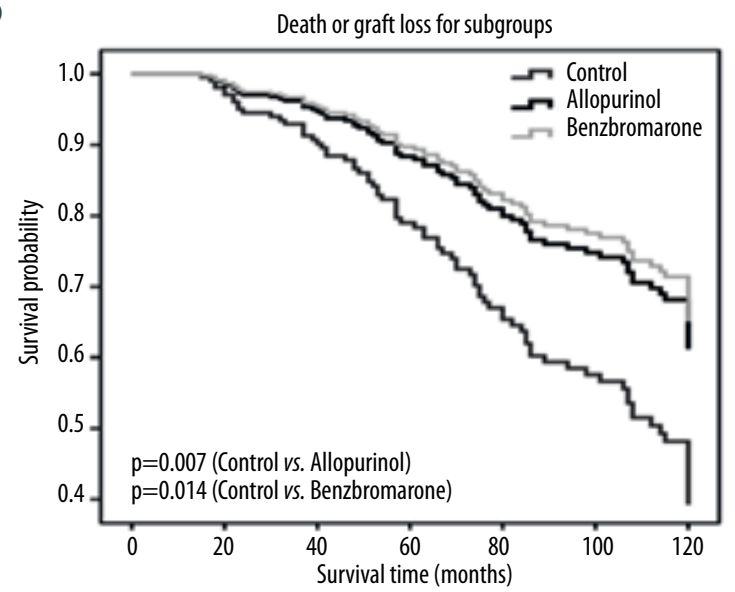

Figure 4. Cox regression analysis survival curves adjusted for age, sex, and eGFR at 12 months for (A) the combined endpoint of death or graft loss, (B) death from any cause, (C) graft loss, and (D) the combined endpoint (graft loss or death) for subgroups (allopurinol or benzbromarone).

\section{Discussion}

The present retrospective analysis presents the first data on the impact of treatment of asymptomatic hyperuricemia on graft function and survival in renal transplant recipients. Treatment led to improved patient and renal graft survival and a benefit in eGFR during 10-year follow-up. These findings are in line with recent reports suggesting that hyperuricemia in renal transplant recipients constitutes an independent risk factor for allograft dysfunction $[13,14]$.

To date, there are no data on the effects of treatment of asymptomatic hyperuricemia in renal transplant recipients. The effect of allopurinol in renal transplant recipients with symptomatic hyperuricemia (gout) was recently investigated in 56 patients. In accordance with our findings, patients treated with allopurinol had a benefit in eGFR during a short follow-up of 3 years [15]. In contrast to our study, however, there was no significant increase in graft survival. Since the effect of hyperuricemia on graft dysfunction depends on the duration of exposure, the follow-up period of 3 years may have been too short and the study size too small to detect a significant difference in survival rates [16]. Interestingly, we found no improved graft or patient survival. Analogous findings have been reported in the non-transplant population: 113 hyperuricemic patients with CKD were randomly assigned to allopurinol or placebo. Treatment with allopurinol was not only associated with a GFR-saving effect but also a reduction in cardiovascular events [6]. Accordingly, in a prospective Scandinavian study including 1423 men, higher levels of SUA were associated with higher cardiovascular mortality even after adjustment for individual risk factors [17]. As a possible explanation, allopurinol was found to reduce left ventricular mass and to improve endothelial function in patients with CKD [18]. By comparing patients with low $(<7 \mathrm{mg} / \mathrm{dl})$ and high $(\geq 7 \mathrm{mg} / \mathrm{dl})$ SUA who were not treated at any time point, we found that 

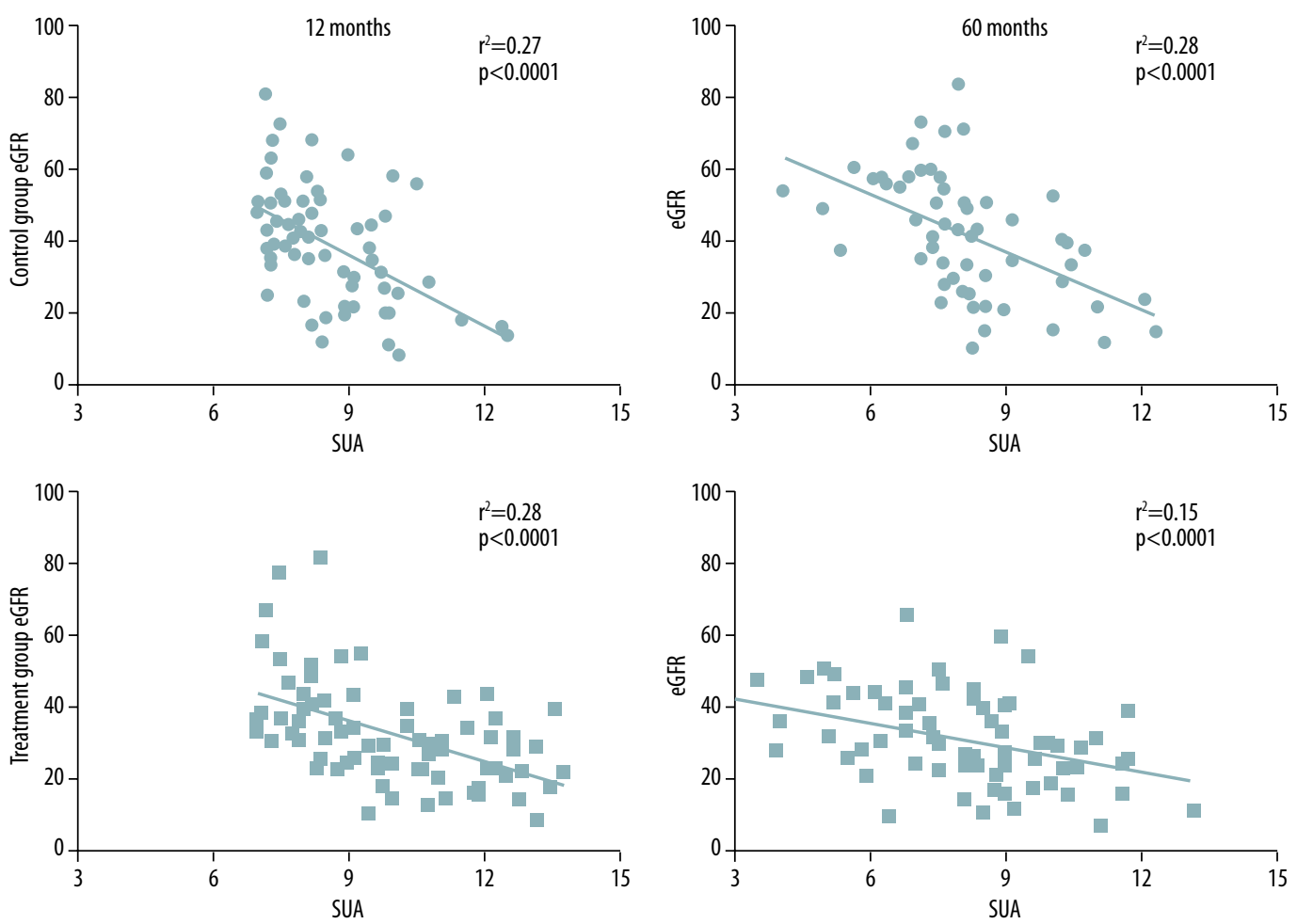

Figure 5. Relationships between eGFR and serum uric acid (SUA) at 12 and 60 months in both groups. A significant inverse correlation between eGFR (in $\mathrm{ml} / \mathrm{min} / 1.73 \mathrm{~m}^{2}$ ) and SUA (in $\mathrm{mg} / \mathrm{dl}$ ) was found at each time point. Only patients who were alive at 60 months with functioning renal graft were considered for analysis. In the treatment group, the slope of the regression tended to be flatter at 60 months compared to 12 months ( $p=0.09)$.

lower SUA is correlated with improved survival and graft function (Figure 2).

In accordance with the benefit in graft survival, treatment of asymptomatic hyperuricemia was associated with a reduction in the annual eGFR $(\triangle G F R)$ decrease after 10 years of followup. This GFR-saving effect of SUA-lowering therapy has been reported in the non-transplant population as well: in patients with chronic kidney disease, allopurinol attenuated the progression of renal dysfunction [6]. Recently, Levy et al. demonstrated that a reduction of SUA to $<6 \mathrm{mg} / \mathrm{dl}$ reduced the number of predefined renal endpoints (decrease of GFR $>30 \%$ or decrease to $\leq 15 \mathrm{ml} / \mathrm{min}$, initiation of dialysis therapy) by $37 \%$ [19]. The discussion on the underlying mechanisms remains rather speculative: uric acid leads to intrarenal vasoconstriction [20] and has proinflammatory properties [21]. Therefore, the GFRsaving effect may be explained by both hemodynamic and antiinflammatory mechanisms. The majority of trials were performed using allopurinol to treat hyperuricemia. Allopurinol, a xanthine oxidase inhibitor, has some pleiotropic effects. Besides the reduction of SUA, inhibition of xanthine oxidase leads to a reduction of reactive oxygen species (ROS), including vascular oxidative stress [22]. Therefore, the question arises of whether the observed benefit in patient and graft survival is mediated by the reduction of SUA or by the drug-related pleiotropic effects of allopurinol. Interestingly, the effects of allopurinol and benzbromarone on survival rates and eGFR were identical in our study (Figure 4D). Hence, the observed benefits were probably primarily mediated by the reduction of SUA itself. However, the small number of patients in each group ( $n=52$ for allopurinol and $n=37$ for benzbromarone) prevents us from being able to provide a final answer to this question.

As described above, there is an ongoing controversy on whether the inverse relationship between SUA and GFR shows a causal role of hyperuricemia in CKD. In accordance with previous trials $[5,23]$, we also found an inverse correlation of SUA and eGFR, as illustrated by the regression line with downward shift in Figure 2. The correlation was independent of the intake of medication for hyperuricemia. If SUA was only a result of a lower eGFR, the slope of the downward regression line in Figure 5 would remain stable between the 2 time points. In the absence of a known causal relationship, the age-dependent decline of eGFR shows as a downward shift of the regression line without a change of its slope. In a recent trial aiming to shed light on the role of SUA as a cause of renal dysfunction, the slope 

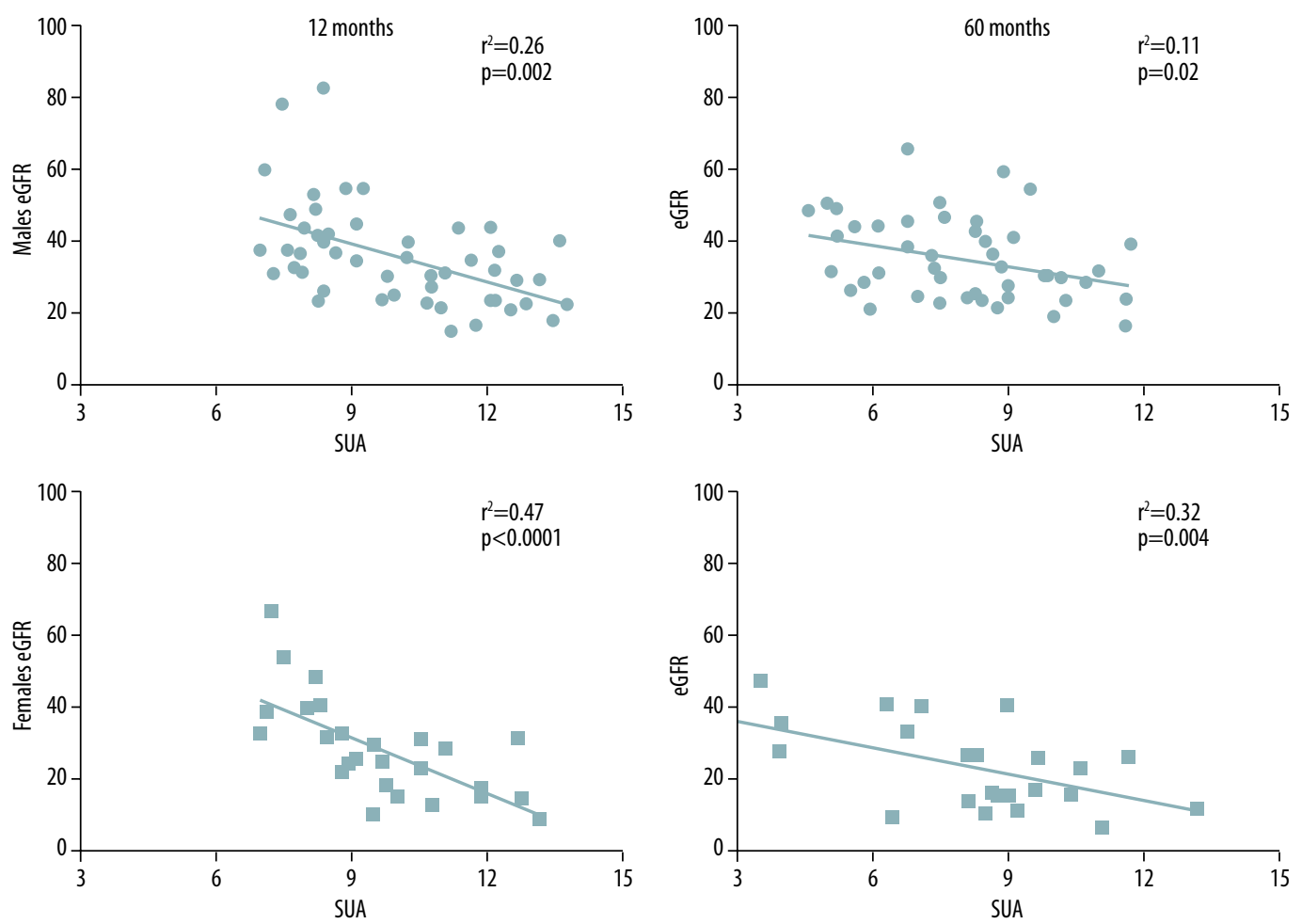

Figure 6. Relationships between eGFR and serum uric acid (SUA) at 12 and 60 months separately for males and females in the treatment group. All patients had a functioning renal graft at 60 months. The slope of the lines in the female group becomes flatter at 60 months, indicating an eGFR-sparing effect of the reduction of SUA (eGFR=-5.22×SUA+78.9 at 12 months versus eGFR $=-2.42 \times S U A+43.5$ at 60 months, $p=0.04$ ). In males, no significant change of the slope was seen between the 2 time points $(p=n s)$. Regression lines for significant correlations are shown in panels.

of the regression line was significantly steeper after a period of 5 years in subjects without established kidney or cardiovascular diseases [5]. This change to a steeper line within 5 years indicates that patients with lower eGFR values also have lower SUA concentrations. Therefore, the authors concluded that SUA plays a causal role in the decline of eGFR. In accordance with these results, in the present study we demonstrate that a drug-induced reduction of SUA tends to flatten the slope of the regression line at 60 months (Figure $5, p=0.09$ ). This finding provides further evidence for the GFR-saving effect of lowering SUA. This trend, seen in the entire treatment group, was statistically significant in females ( $p=0.04$, Figure 6 ). Interestingly, recent reports indicate that women are more susceptible to hyperuricemia-induced reductions of GFR: lower levels of SUA, compared to men, led to increased renal impairment [23] and microalbuminuria [24]. In a study by Akasaka, an analogous effect was seen in the female group, in which SUA was associated with a stronger decrease in eGFR [5].

A prospective trial in adolescents recently revealed antihypertensive effects of lowering SUA by use of allopurinol [25]. In the present study we did not demonstrate any effect on blood pressure. It remains unknown whether a potential effect on blood pressure was masked by changes in antihypertensive treatment, or whether the prohypertensive effects of immunosuppressants are less susceptible to SUA-lowering therapy.

Hyperuricemia itself is not a disease; therefore, the current American [3] and European [26] guidelines do not recommend treatment of asymptomatic hyperuricemia. Since there are no separate guidelines for renal transplant recipients, these recommendations are usually applied to these patients in daily clinical practice. Renal transplant recipients, however, have an extraordinarily increased cardiovascular risk. Moreover, conservation of residual renal function is of outstanding relevance for these patients. Every available method to slow the progression of allograft dysfunction should be strictly used. These measures include optimization of immunosuppression, blood pressure, and the metabolic profile, including glucose, lipids, and acidbase balance. Our results suggest that treatment of hyperuricemia should be considered as a tool for conservation of graft function and reduction of cardiovascular risk. A prospective randomized controlled trial is desirable in this context but is unlikely to be performed in the near future. Interestingly, the 
Japanese Guideline for the Management of Hyperuricemia and Gout [27] constitutes an exception among the current guidelines and already recommends treatment of asymptomatic hyperuricemia in patients with cardiovascular comorbidities.

The present study has several limitations. First, patients were recruited retrospectively; therefore, the existence of selection bias cannot be finally excluded in both groups. Second, the decision to treat or not to treat hyperuricemia was personally taken by the nephrologist of the outpatient clinic; therefore, assignment to the 2 groups was based on a subjective decision. Groups, however, were homogenous for graft function and cardiovascular risk profile. In addition, we adjusted groups for eGFR, age, and sex.

\section{References:}

1. Lin HY, Rocher LL, McQuillan MA et al: Cyclosporine-induced hyperuricemia and gout. N Engl J Med, 1989; 321(5): 287-92

2. Akalin E, Ganeshan SV, Winston J, Muntner P: Hyperuricemia is associated with the development of the composite outcomes of new cardiovascular events and chronic allograft nephropathy. Transplantation, 2008; 86(5): 652-58

3. Khanna D, Fitzgerald JD, Khanna PP et al: 2012 American College of Rheumatology guidelines for management of gout. Part 1: systematic nonpharmacologic and pharmacologic therapeutic approaches to hyperuricemia. Arthritis Care Res, 2012; 64(10): 1431-46

4. Kang $\mathrm{DH}$, Nakagawa T, Feng $\mathrm{L}$ et al: A role for uric acid in the progression of renal disease. J Am Soc Nephrol, 2002; 13(12): 2888-97

5. Akasaka $H$, Yoshida $H$, Takizawa $H$ et al: The impact of elevation of serum uric acid level on the natural history of glomerular filtration rate (GFR) and its sex difference. Nephrol Dial Transplant, 2014; 29(10): 1932-39

6. Goicoechea M, de Vinuesa SG, Verdalles $U$ et al: Effect of allopurinol in chronic kidney disease progression and cardiovascular risk. Clin J Am Soc Nephrol, 2010; 5(8): 1388-93

7. Fang J, Alderman MH: Serum uric acid and cardiovascular mortality the NHANES I epidemiologic follow-up study, 1971-1992. National Health and Nutrition Examination Survey. JAMA, 2000; 283(18): 2404-10

8. Culleton BF, Larson MG, Kannel WB, Levy D: Serum uric acid and risk for cardiovascular disease and death: the Framingham Heart Study. Ann Intern Med, 1999; 131(1): 7-13

9. Neal DA, Tom BD, Gimson AE et al: Hyperuricemia, gout, and renal func tion after liver transplantation. Transplantation, 2001; 72(10): 1689-91

10. Neogi T: Clinical practice. Gout. N Engl J Med, 2011; 364(5): 443-52

11. Marcen R, Morales JM, Fernandez-Rodriguez A et al: Long-term graft function changes in kidney transplant recipients. NDT Plus, 2010; 3(Suppl.2): ii2-ii8

12. Pestana JO, Grinyo JM, Vanrenterghem Y et al: Three-year outcomes from BENEFIT-EXT: a phase III study of belatacept versus cyclosporine in recipients of extended criteria donor kidneys. Am J Transplant, 2012; 12(3): 630-39

13. Huang $\mathrm{Y}$, Li YL, Huang $\mathrm{H}$ et al: Effects of hyperuricemia on renal function of renal transplant recipients: A systematic review and meta-analysis of cohort studies. PLoS One, 2012; 7(6): e39457

14. Weng SC, Shu KH, Tarng DC et al: Uric acid is highly associated with kidney allograft survival in a time-varying analysis. Transplant Proc, 2014; 46(2): 505-10

\section{Conclusions}

Treatment of asymptomatic hyperuricemia by allopurinol or benzbromarone led to improved patient and graft survival in the present cohort of renal allograft recipients. Lowering SUA resulted in a slower deterioration of graft function. The present analysis may provide arguments to treat asymptomatic hyperuricemia after kidney transplantation. However, randomized controlled trials are needed to confirm these results.

\section{Statement}

No financial support/interest to declare.

15. Osadchuk L, Bashir MH, Tangirala B et al: Effect of allopurinol on slowing allograft functional decline in kidney transplant recipients. Exp Clin Transplant, 2014; 12(3): 190-94

16. Min SI, Yun IJ, Kang JM et al: Moderate-to-severe early-onset hyperuricaemia: A prognostic marker of long-term kidney transplant outcome. Nephrol Dial Transplant, 2009; 24(8): 2584-90

17. Niskanen LK, Laaksonen DE, Nyyssonen $\mathrm{K}$ et al. Uric acid level as a risk factor for cardiovascular and all-cause mortality in middle-aged men: A prospective cohort study. Arch Intern Med, 2004; 164(14): 1546-51

18. Kao MP, Ang DS, Gandy SJ et al: Allopurinol benefits left ventricular mass and endothelial dysfunction in chronic kidney disease. J Am Soc Nephrol, 2011; 22(7): 1382-89

19. Levy GD, Rashid N, Niu F, Cheetham TC: Effect of urate-lowering therapies on renal disease progression in patients with hyperuricemia. J Rheumatol, 2014; 41(5): 955-62

20. Sanchez-Lozada LG, Tapia E, Santamaria J et al: Mild hyperuricemia induces vasoconstriction and maintains glomerular hypertension in normal and remnant kidney rats. Kidney Int, 2005; 67(1): 237-47

21. Krishnan E: Inflammation, oxidative stress and lipids: The risk triad for atherosclerosis in gout. Rheumatology, 2010; 49(7): 1229-38

22. George J, Carr E, Davies J, Belch JJ, Struthers A: High-dose allopurinol improves endothelial function by profoundly reducing vascular oxidative stress and not by lowering uric acid. Circulation, 2006; 114(23): 2508-16

23. Kamei K, Konta T, Hirayama A et al: A slight increase within the normal range of serum uric acid and the decline in renal function: Association in a community-based population. Nephrol Dial Transplant, 2014; 29(12): 2286-92

24. Lee JE, Kim YG, Choi YH et al: Serum uric acid is associated with microalbuminuria in prehypertension. Hypertension, 2006; 47(5): 962-67

25. Feig DI, Soletsky B, Johnson RJ: Effect of allopurinol on blood pressure of adolescents with newly diagnosed essential hypertension: $A$ randomized trial. JAMA, 2008; 300(8): 924-32

26. Zhang W, Doherty $M$, Bardin T et al: EULAR evidence based recommendations for gout. Part II: Management. Report of a task force of the EULAR Standing Committee for International Clinical Studies Including Therapeutics (ESCISIT). Ann Rheum Dis, 2006; 65(10): 1312-24

27. Yamanaka $\mathrm{H}$; Japanese Society of Gout and Nucleic Acid Metabolism: Japanese guideline for the management of hyperuricemia and gout: Second edition. Nucleosides Nucleotides Nucleic Acids, 2011; 30(12): 1018-29 